\title{
Telomere dysfunction is associated to dark-induced coral bleaching in the reef coral Stylophora pistillata
}

Eric Gilson ${ }^{1}$, Alice Rouan ${ }^{2}$, Sylvie Tambutté ${ }^{3}$, Didier Zoccola ${ }^{2}$, Melanie POUSSE ${ }^{2}$, Eric Tambutté $^{3}$, Nadir Djerbi ${ }^{2}$, Laura Capasso ${ }^{2}$, and William Zozaya ${ }^{2}$

${ }^{1}$ IRCAN

${ }^{2}$ Affiliation not available

${ }^{3}$ Centre Scientifique de Monaco

April 20, 2021

\begin{abstract}
Telomere DNA length is a complex trait controlled both by multiple loci and environmental factors. Even though the use of telomere DNA length measurement, as a method of assessing stress accumulation and predicting how this will influence survival, is currently being studied in numerous human cohort studies, the importance of telomere length for stress response in ecological studies remains at its infancy. Here, we investigated the telomere changes occurring in the symbiotic coral Stylophora pistillata that has experienced a continuous darkness over six months. This stress condition led to the loss of its symbionts, as what is also observed when bleaching occurs in the field at a large-scale due to climate changes and anthropogenic activities, threatening the worldwide reef ecosystem. We found that the continuous darkness condition was associated with telomere DNA length shortening and a downregulation of the expression of the telomere-associated protein Pot2. These results pave the way for future studies on the role of telomere in coral stress response and the importance of telomere dysregulation in endangered coral species.
\end{abstract}

\section{Telomere dysfunction is associated}

to dark-induced coral bleaching in the reef coralStylophora pistillata

Alice Rouan ${ }^{1}$, Mélanie Pousse ${ }^{1}$, Eric Tambutté ${ }^{2}$, Nadir Djerbi $^{1}$, William Zozaya ${ }^{1}$, Laura Capasso ${ }^{2,3}$, Didier Zoccola $^{2}$, Sylvie Tambutté ${ }^{*}$, Eric Gilson ${ }^{1,4^{*}}$

${ }^{1}$ Université Côte d'Azur-CNRS-Inserm, IRCAN, Nice, France.

2 Department of Marine Biology, Centre Scientifique de Monaco, Monte Carlo, Principality of Monaco.

${ }^{3}$ Sorbonne Université, Collège Doctoral, F-75005 Paris, France

${ }^{4}$ Department of Medical Genetics, CHU, Nice, France.

* Corresponding authors : Eric.GILSON@univ-cotedazur.fr, stambutte@centrescientifique.mc

\begin{abstract}
Telomere DNA length is a complex trait controlled both by multiple loci and environmental factors. Even though the use of telomere DNA length measurement, as a method of assessing stress accumulation and predicting how this will influence survival, is currently being studied in numerous human cohort studies, the importance of telomere length for stress response in ecological studies remains at its infancy. Here, we investigated the telomere changes occurring in the symbiotic coralStylophora pistillata that has experienced
\end{abstract}


a continuous darkness over six months. This stress condition led to the loss of its symbionts, as what is also observed when bleaching occurs in the field at a large-scale due to climate changes and anthropogenic activities, threatening the worldwide reef ecosystem. We found that the continuous darkness condition was associated with telomere DNA length shortening and a downregulation of the expression of the telomereassociated protein Pot2. These results pave the way for future studies on the role of telomere in coral stress response and the importance of telomere dysregulation in endangered coral species.

\section{Introduction}

Scleractinian corals are metazoans that successfully built complex ecosystems, the coral reefs, which are among the most important biodiversity hotspots of the planet. However, all over the world, coral reefs are under threat due to global climate change and anthropogenic activities (Hughes et al. 2018b). These stressors can cause imbalances in the community of endosymbiotic partners hosted by corals such as viruses, bacteria, fungi and most notably, members of the algal dinoflagellate family Symbiodiniaceae. The breakdown of this intricate symbiosis leads to the loss of the intracellular symbionts and/or their pigments, a process that is called bleaching (Downs et al. 2013; Sully et al. 2019; Downs et al. 2009; Danovaro et al. 2008; Kushmaro et al. 1996; Rosenberg et al. 2009). Corals can either recover from bleaching by acquiring new symbionts or die in case of prolonged or repeated stresses. Of note, bleaching can be triggered in the laboratory under different experimental set-up (increases in temperature or light intensity, continuous darkness, hyposalinity, chemical pollutants and pathogen infection) but if food is provided, the corals can survive for long time periods even though different physiological processes can be affected (body mass loss, stop of calcification, growth arrest and polyp mortality) (Lyndby et al. 2020; Gardner et al. 2017).

In order to mitigate coral loss, there is currently an urgent need to understand the mechanisms involved in the response of corals to stress and among them, the pathways maintaining genome stability are essential. An accurate response to DNA damages caused by environmental stressors (radiation, heat, pollutants, nutrient fluctuations...) as well as normal metabolic processes (such as replication, respiration and, in the case of symbiotic corals, photosynthetic activity leading to daily oxidative switches), requires efficient DNA damage checkpoints, DNA repair and survival pathways to maintain genome stability and health (López-Otín and Kroemer, 2021). Therefore, the efficiency of DNA repair mechanisms is one of the main regulators of stress resistance and longevity in many organisms (Ma et al.,2016; Tian et al., 2019). However, aside from antioxidant defenses (Furla et al., 2005) and UV DNA repair (Reef et al., 2009) and recent report on increased levels of phosphorylated H2AX (a marker of DNA damage response) (Rodriguez-Casariego et al., 2018) and Rad51 (a DNA double strand break repair protein) (Maor-Landaw and Levy, 2016) during heat stress, our knowledge on the role of genome maintenance pathways in environmental tolerance in corals remains limited.

Among the genome stability mechanisms, the chromosome ends, or telomeres, play a key role in stress response. Telomeres are composed of DNA repeats, (TTAGGG)n in most metazoans (Traut et al. 2007), bound by a protective protein complex, the shelterin, which protects the chromosome against unwanted DNA damage response (de Lange 2005). In humans, the shelterin complex is made of six proteins (TRF1, TRF2, TIN2, TPP1, POT1 and RAP1) (Giraud-Panis et al. 2013). TRF1 and TRF2 (Telomeric-repeat binding factor 1 and 2) directly bind the duplex telomeric DNA repeats, POT1 (Protection of Telomere 1) binds the single stranded telomeric DNA forming the 3' overhangs of chromosomal DNA, while TIN2 and TPP1 bridge the double and single stranded parts of telomeric DNA. In addition, RAP1 is associated with TRF2. The telomerase holoenzyme, comprising the reverse transcriptase subunit TERT and its RNA template, is able to maintain telomere length during DNA replication (Gilson and Geli 2007). Functional telomeres protect chromosomes against instability and senescence and are also involved in a wide range of important processes such as inflammation, immunity, energetic metabolism and mitochondrial integrity, stemness as well as cellular differentiation (Ye et al., 2014). Changes in telomere structure occur during development, stress response and aging. In general, exposure to unfavorable environments leads to accelerated telomere shortening (Young et al., 2017). In birds, telomere DNA length can predict key life-history traits like growth, reproduction and lifespan (Monaghan, 2014). Thus, it was proposed that change in telomere DNA length is 
an adaptive strategy based on life-history regulation and environmental adaptation (Young 2018) and that telomere DNA length is a useful biomarker for past stress (Bateson, 2016).

Here we investigated the impact on telomere structure of continuous darkness stress (six months) in the model coral Stylophora pistillata (Esper, 1797). Notably, we observed that this stress condition leads to telomere DNA length shortening as well as a downregulation of the expression of a coral ortholog of a shelterin subunit. Since this stress is associated with the bleaching of the coral colony (Supplementary Figure 2), we discuss the possibility that the telomere response to continuous darkness is a consequence of the bleaching state.

\section{Material and methods}

\section{Sampling}

Colonies of the tropical coral S. pistillata were exposed to long-term darkness in an experimental aquarium setup for a long term exposure of six months. Briefly, coral fragments were kept in aquaria supplied with Mediterranean seawater (exchange rate of $70 \%$ per hour) at a salinity of $38 \mathrm{~g} \mathrm{liter}^{-1}$, temperature of $25 \mathrm{degC}$, $\mathrm{pH}_{\mathrm{T}} 7.94+-0.02$ and for the control condition at an irradiance of $230 \mu \mathrm{mol}$ photons $\mathrm{m}^{-2} \mathrm{~s}^{-1}$ on a $12: 12$ photoperiod. Both dark and control conditions corals were fed daily with frozen rotifers and twice a week with live artemia nauplii. The first experiment D1 was conducted from July 2018 to January 2019 were a part of the S1 colony was separated and put in dark condition, 6 branches of the bleached colony were sampled and 5 of the control one, the second experiment D2 was conducted from June 2019 to December 2019 were a different $S$. pistillata colony was divided in 2 part, each one kept in either of the two experimental condition, 4 branches from each colony part were sampled.

\section{DNA extraction}

DNA was extracted on the first 3-4 cm of a colony branch. All steps until cell lysis were done on ice. The fresh coral tissue was removed from the skeleton using the "air_brush" technique in 50mL of extraction buffer ( $\mathrm{NaCl} 550 \mathrm{mM}$, EDTA 0,2M (Tambutté et al., 2007)) in a Whirl-Pak bag (Nasco) under the hood, then centrifuged for 10 minutes at 5 000g (Beckman Coulter, Avanti J-E centrifuge, rotor JA-18) at $4^{\circ} \mathrm{C}$. The tissue pellet was kept on ice to perform high molecular weight DNA extraction following the Midi Kit Cell and Blood culture (QIAGEN). Briefly, cell lysis was carried out for 2 hours at $50^{\circ} \mathrm{C}$ after adding 19 $\mathrm{mL}$ of $\mathrm{G} 2$ buffer $(800 \mathrm{mM}$ guanidine $\mathrm{HCl} ; 30 \mathrm{mM}$ Tris. $\mathrm{Cl}$, pH $8.0 ; 30 \mathrm{mM}$ EDTA, $\mathrm{pH} 8.0$; 5\% Tween-20 ; 0,5\% Triton X-100, QIAGEN) with $190 \mathrm{~mL}$ RNase A (ThermoFisher, ref : $12091039,20 \mathrm{mg} / \mathrm{mL}$ ) and 400 $\mathrm{mL}$ of protease (QIAGEN, $1 \mathrm{UA} / \mathrm{mL}$ ) to the tissue pellets. Supernatant was poured in the anionic column after a 5 min centrifuge at $5000 \mathrm{~g}$ and $4^{\circ} \mathrm{C}$, columns were washed following the kit instructions, DNA was eluted with $5 \mathrm{~mL}$ of warm QF buffer (1.25 M NaCl; $50 \mathrm{mM}$ Tris.Cl, $\mathrm{pH} 8.5 ; 15 \%$ isopropanol, QIAGEN). Precipitation was performed adding $3.5 \mathrm{~mL}$ of 2-propanol (Sigma), after mixing by inverting, tubes were stored overnight at $4^{\circ} \mathrm{C}$. Two washing steps were performed adding $1 \mathrm{~mL}$ of $70 \%$ ethanol (sigma) to DNA pellets after $30 \mathrm{~min}$ centrifuge at $15000 \mathrm{~g}$ and $4^{\circ} \mathrm{C}$ before adding $200 \mathrm{~mL}$ of TE buffer (Tris-Cl pH8 10mM, EDTA $1 \mathrm{mM}$ ) for long-term storage. Pellets were resuspended at $50^{\circ} \mathrm{C}$ for 1 hour. To test DNA quality and quantity both Nanodrop (ThermoFisher) and agarose gel were used, respectively $1 \mathrm{~mL}$ of DNA and 1,5 mL of loading buffer (New England Biolabs, 6X) in a final volume of $10 \mathrm{~mL}$ were loaded in a $1.2 \%$ agarose gel run for 30 minutes at 100V in Tris-Borate-EDTA (TBE) 1X. Agarose gel was incubated for 10 minutes in 4 $\mathrm{mg} / \mathrm{mL}$ ethidium bromide and DNA was revealed under UV (GelDoc Transilluminator, BioRad), the average of three measurements was used. A diluted aliquot was prepared for enzymatic digestion before storing the stock DNA at $-20^{\circ} \mathrm{C}$.

Telomere Restriction Fragment assay:

Dilution and DNA digestion

To digest non telomeric DNA sequences, we added 60U HinfI (R0155M, New England Biolabs) and 60U RsaI (R4374, Promega) to of 2,5 mg of DNA diluted in mqH2O and "CutSmart Buffer" 1X (New England 
Biolabs) in a final volume of $40 \mathrm{~mL}$, left overnight at $37^{\circ} \mathrm{C}$.

\section{Southern Blot}

We measured telomere length using the Telomeric Restriction Fragment assay (TRF) using the Southern blotting procedure (Herbert et al., 2003) digested DNA was loaded on a 1,2\% agarose gel for a $3 \mathrm{~h} 30$ run in TBE $0.5 \mathrm{X}$ at 9 volts $/ \mathrm{cm}$ in a CHEF-DR-II (BioRad). The run timing makes it suitable for 2 runs a day. After a 5 min wash in $\mathrm{mQ} \mathrm{H} 2 \mathrm{O}$ agarose gel was stained in Ethidium bromide bath $(4 \mathrm{mg} / \mathrm{mL})$ under agitation for 30min and imaged the gel (Typhoon, GE Healthcare, Fluorescence, Method=Alexa Fluor 555, Laser $=532 \mathrm{~nm}, \mathrm{PMT}=\mathrm{ch} .1,700 \mathrm{~V}$, Resolution $=50 \mu \mathrm{m})$. Denaturation was done in $(1 \mathrm{M} \mathrm{NaCl} ; 0.5 \mathrm{M} \mathrm{NaOH})$ for two incubations of $20 \mathrm{~min}$ followed by a neutralization step with two $20 \mathrm{~min}$ incubation in $1 \mathrm{M}$ ammonium acetate. The DNA was transferred to a Hybond $\mathrm{N}^{+}$membrane (GE Healthcare) overnight in SSC (20X). DNA was cross-linked on the membrane using the Ultraviolet Crosslinker (UVP) at $1200 \times 100 \mu$ Joules. Membrane was gently washed in $\mathrm{mQH}_{2} \mathrm{O}$ before storage at room temperature for $(12 \mathrm{~h}$ up to $48 \mathrm{~h}$ ) before $1 \mathrm{~h}$ pre-hybridization in Denhardt's buffer $(10 \mathrm{X})$ at $40^{\circ} \mathrm{C}$. We prepared stocks of Denhardt's $100 \mathrm{X}$ buffer $(2 \%$ Ficoll 400, 2\% Bovine Serum Albumine, $2 \%$ Polyvinylpyrrolidone) filtered $(0.2 \mu \mathrm{m})$ and stored at $-20^{\circ} \mathrm{C}$.

Probe synthesis

We prepared probe template 40uM stocks annealing two primers per probe. Probe targeting (TTAGGG) sequence will be called T2 and the one targeting (TTTAGGG) sequence will be referred to as T3. We mixed 25 of Primer $\mathrm{F}$ and 25 of Primer R (Primer T2AG3_F : GgGTtAGgGTTAGGgtTagGGaAA and T2AG3_R : TTTCCCTAACCCTAA, PrimerT3AG3_F : GGGTTTAGGGTTTAGGGTTTAGGGAAA and T3AG3_R : TTTCCCTAAACCCTAAA) with STE 5X buffer (Tris 50mM pH8, NaCl 250mM, EDTA $5 \mathrm{mM}$ ) and heat it to $95^{\circ} \mathrm{C}$ to therefore gradually let it cool down to room temperature before being stored at $-20^{\circ} \mathrm{C}$. We mixed on ice 2.5 of dATP $(10 \mathrm{mM})$ and 2.5 of dTTP $(10 \mathrm{mM}$, New England Biolabs, R0192) with 3of NEB 2 buffer (10X), 15of mQH2O, 5 of gamma-dCTP32 and 1 Klenow (3'->5' exo-) (M0212S, NEB) incubated in a thermocycler (ThermoFisher Scientific) for a $\left(30 \mathrm{~min} 25^{\circ} \mathrm{C}, 5 \mathrm{~min} 98^{\circ} \mathrm{C}, 5 \mathrm{~min} 25^{\circ} \mathrm{C}\right.$ ) program. Then we purified the probes following the ProbeQuant G50-micro columns (GE, Healthcare) instructions. Probes were denatured at $95^{\circ} \mathrm{C}$ for 5 min and used or stored at $-20^{\circ} \mathrm{C}$.

\section{Hybridization}

Membranes were pr hybridized for $1 \mathrm{~h}$ at $40^{\circ} \mathrm{C}$ in hybridization Denhardt's buffer (10X). We added $5 \mu \mathrm{L}$ of denatured T3 probes to $15 \mathrm{~mL}$ of hybridization buffer for an overnight hybridization at $40^{\circ} \mathrm{C}$. Washing steps included a $2 \times 20 \mathrm{~min}$ wash in $2 \mathrm{X}$ SSC, a $30 \mathrm{~min}$ step in (2X SSC, SDS 0,2\%) and a final $\mathrm{H} 2 \mathrm{O}$ washing step. Membrane was carefully wrapped in plastic sarran film and applied to a phosphor-screen (brand) for $60 \mathrm{~h}$. We imaged the phospho-screen on the Typhoon (GE Healthcare, Phosphorimaging, Method=[Phosphor], Laser $=635 \mathrm{~nm}, \mathrm{PMT}=\mathrm{ch} .1,1000 \mathrm{~V}$, Resolution $=50 \mu \mathrm{m}$ ), the screen was flashed (brand of device). Membrane was stripped with $\mathrm{NaOH} 0,4 \mathrm{M}$ for $30 \mathrm{~min}$ at $42^{\circ} \mathrm{C}$, then for 30min in (SDS 1\%, SSC 0.1X, Tris-HCl pH7.4 $1 \mathrm{M})$ to remove the T3 probe. To control the stripping, membrane we exposed the membrane to a flashed phosphor-screen for $12 \mathrm{~h}$. The screen was imaged as described above, in the absence of signal the membrane was pre hybridized for $1 \mathrm{~h}$ and hybridized overnight at $45^{\circ} \mathrm{C}$ with the $\mathrm{T} 2$ probe, otherwise the membrane was stripped again until no signal was imaged after $12 \mathrm{~h}$ of exposure. Washing steps and imaging were carried out as described above with a $24 \mathrm{~h}$ exposure of the phosphor-screen.

\section{Image analysis}

Telomere Restriction Fragment (TRF) images signal was extracted using ImageQuant (GE Healthcare) 1D gel analysis mode and manual lane creation. Ladders signal intensity were extracted from Ethidium bromide gel images, setting the lane upper limit at the gel wells bottom. Pixel position of ladder peaks were manually reported. Host and symbiont telomere signals were extracted from the phospho screen, exposed to radioactive labelled membrane, images setting sample lane upper limit at the top of the membrane. Efficiency of stripping step between the two probes hybridization was assessed imaging phospho screens overnight exposed to stripped membranes. 


\section{Telomere Length measurements}

Single lane intensity files were fused in RStudio. Ladder peaks exact position was extracted in R, searching for the maximum intensity in a 10-pixel perimeter around the manually reported peaks. Fitted linear model coefficients $(\mathrm{a}, \mathrm{b})$ of $\log 2$ ladder size $(\mathrm{kb})$ against peak pixel position were calculated for high molecular weight (48.5-15kb) and low molecular weight (10-1kb) using the $l m$ function of "stats" R package. Coefficients were used to transform samples intensity scale from pixel to base pair (bp) () in Excel using the high molecular weight coefficient for the upper part and the low molecular coefficient for the lower one. Depending on their position on the membrane samples were divided in left, right and middle to be scaled to the closest ladder. Intensity signals were imported in $\mathrm{R}$ using the read_excel from "readxl" package, background correction was automatically computed to level the signal by subtracting to each position the minimal intensity. Intensity was normalized by the size to avoid probe number hybridization bias, intensity above $2 \mathrm{~kb}$ were discarded to avoid genomic noise, interstitial telomeric sequence noise and normalization bias $(<1 \mathrm{~kb})$. Ponderate telomere mean was calculated as in ( $\mathrm{Li}$ and de Lange 2003), median, quartiles, intensity signal, smoothed signal and telomere measurements were plotted for each sample using the ggplotfunction from "ggplot2" package and measurements were saved ascsv files.

Transcriptome

\section{RNA extraction}

Coral pieces of approximately $2 \mathrm{~cm}$ were sampled just below the coral piece dedicated to DNA extraction, at least $4 \mathrm{~cm}$ from the apex of the branch. Samples were flash frozen in liquid nitrogen in WhirlPack bags, and then crushed in small pieces with a press and fragments were put in 600uL of lysis buffer RLT in PowerBeads Glass 0.1mm (Qiagen), samples were lysed in Bead Beating instrument for 4 minutes with 30 cycles/s. Samples were spin at max speed for 3 minutes and the $600 \mathrm{uL}$ of supernatant was transferred to a fresh tube. Add 600uL (1V) of cold EtOH $70 \%$ to the column (Qiagen, RNeasy Mini Kit), gently mixed by pipetting, immediately apply $600 \mathrm{uL}$ on the column and centrifuge $15 \mathrm{~s}$ at $8000 \mathrm{~g}(10000 \mathrm{rpm})$, discard the flow-through, repeat this step. Add $350 \mathrm{uL}$ of RWI buffer to the column and centrifuge $15 \mathrm{~s}$ at $8000 \mathrm{~g}$ (10 $000 \mathrm{rpm}$ ), discard the flow-through. Take a DNAse, Rnase free, (Qiagen, 79254) stored at $-20^{\circ} \mathrm{C}$, add $70 \mathrm{ul}$ of buffer RDD $4^{\circ} \mathrm{C}$ and apply to the column. Leave the column at RT for $15 \mathrm{~min}$ before adding 350 $\mathrm{uL}$ of RWI buffer and centrifuge $15 \mathrm{~s}$ at $8000 \mathrm{~g}$ (10 $000 \mathrm{rpm})$, discard the flow-through and add $500 \mathrm{uL}$ of RPE buffer, repeat the centrifugation, add again 500uL of RPE buffer and make a 2 min at $8000 \mathrm{~g}(10000$ $\mathrm{rpm}$ ) centrifuge before discarding the flow-through and putting the column in a clean $1.5 \mathrm{ml}$ tube. Add 30ul of RNase free water to the column and centrifuge for $1 \mathrm{~min}$ at $8000 \mathrm{~g}(10000 \mathrm{rpm})$, keep the tube on ice for further experiment and store at $-80^{\circ} \mathrm{C}$. RNA quantity was measured on a microplate spectrophotometer Epoch (Bioteck) and the quality was assessed by running a 1\% agarose gel labelled with Ethidium Bromide and imaged under UV-exposure in a Fusion Fx7.

\section{RNA sequencing}

Paired-end sequencing (read length: 2x150bp) was performed by NovoGene company using HiSeq sequencer, raw data are available with accession number GSE171268 (https://www.ncbi.nlm.nih.gov/geo/query/acc.cgi?acc=GSE171268). Three biological replicates per condition were performed with roughly 45 million reads per sample. Reads were trimmed using fastp software (Chen et al., 2018). Reads were mapped on the S.pistillatatranscriptome from NCBI (GCF_002571385.1) using Salmon software (v0.11.4) (Patro et al., 2017). The differential genes expression analysis was performed using DESeq2 R package (Love et al., 2014). A cutoff of 0.05 was applied on adjusted p-values, obtained using Benjamini and Hochberg correction.

Ontology and pathway analysis

Gene Ontology (GO) analysis was performed using the "topGO" R package (Alexa et al., 2006). For the cnidarian analysis, all GO terms identified in S.pistillata were used as background. Ingenuity Pathway Analysis (Krämer et al. 2014) (QIAGEN,https://www.qiagenbioinformatics.com/products/ingenuitypathway-analysis) 
was performed on human ortholog genes. Kyoto Encyclopedia of Genes and Genomes (KEGG) pathway analysis was performed using "gage" and "KEGGREST" R packages (Kanehisa, 2000). The human ortholog genes associated with the S.pistillata log2FoldChange values were used to conduct the analysis. Human orthologs were identified using the protein sequences. Blastp (Altschul et al. 1990) on UniProtKB/Swiss-Prot database restricted to Homo sapiens species was performed with a 1e-5 e-value threshold. For each protein, the best hit was selected, based on e-value and bit-score.

Phylogeny

Ortholog sequences of S.pistillata shelterin proteins were curated using PSI-BLAST (Altschul et al, 1997) on the non-redundant $(\mathrm{Nr})$ protein database from the NCBI. The search was restricted to Metazoa group. The retrieved sequences were aligned using MAFFT v7.310 with L-INS-I algorithm (Katoh and Standley, 2013). The multiple alignment was trimmed with TrimAl v1.4 with the gt 0.6 option (Capella-Gutierrez et al., 2009). Then the best substitution matrix was assessed using ModelTest (Darriba et al., 2020). Finally, the trees were built using raxmlGUI 2.0, a graphical interface for the implementation of RAxML Next Generation and the transfer bootstrap expectation branch support (Edler et al., 2020). Tree visualization was done with iToL v6.1 (Letunic and Bork, 2019). Multiple alignment for POT1 and POT2 were wrapped using Alignment Annotator (Gille et al., 2014).

Quantitative Polymerase Chain Reaction (PCR)

Primers were designed using PrimerBlast (Ye et al., 2012) and sequences are available in (Supplementary Table 3). Reverse transcription was carried for 1ug of RNA samples using the Applied Biosystems reverse transcription kit for $1 \mathrm{~h}$ at $37^{\circ} \mathrm{C}$ followed by $5 \mathrm{~min}$ of denaturation at $95^{\circ} \mathrm{C}$. qPCRs were made on cDNAs obtained using ROCHE's Fast universal Sybr Greenmaster (ROX) on a StepOne plus thermocycler (Applied Biosystems). Each sample was triplicated on the 96 wells PCR plate containing the interest gene primers and the two control genes. In addition to the samples from the RNAseq, samples from the same experiment were added to the qPCR, two additional control samples (S1n2 and S1n4) as well as two treated samples (S1Bn2 and S1Bn6).

Statistics

Telomere length measurements were averaged from two independent Telomeric Restriction Fragment assay, homoscedasticity and normal distribution were tested on $\mathrm{R}$ using (fligner.test andshapiro.test functions) and significant differences was calculated using the t_test from the "rstatix" packages with a Bonferroni pvalue adjustement. Significant differences are reported as stars ( (ns) non significant, $\left(^{*}\right) \mathrm{P}<0.05,\left(^{* *}\right) \mathrm{P}<0.01$, $(* * *) \mathrm{P}<0.001$.

\section{Results}

Putative telomere proteins encoded in the Stylophora pistillata genome

To characterize the structure of coral telomeres, we searched in theS. pistillata host genome (Voolstra et al. 2017) for genes encoding putative shelterin subunits as well as the telomerase catalytic subunit TERT. We identified orthologs of four human shelterin subunits (Rap1, Trf1/2, Pot1, Tpp1) and of Tert (Figure 1A-BC-D-E). The coral genome contains two human Pot1 paralogs, similarly to mouse and Caenorhabditis elegans elegans (Raiceset al. 2008), and only one $\operatorname{Trf}$, in agreement with the duplication of the $\operatorname{Trf}$ ancestor in $\operatorname{Trf} 1$ and Trf2that occurred at the base of the chordate lineage (Poulet et al.2012) (Figure 1B). The coral Pot1 protein shares a similar structure with the human Pot1, containing two Oligonucleotide/OligosaccharideBinding (OB) fold domains located in the N-terminal region and a characteristic split OB fold in the Cterminus (Figure1-G, Supplementary Figure 1). The second ortholog of Pot, named Pot2, contains two OB folds skewed to the C-terminal part of the protein. Therefore, the protein composition of coral telomeres is likely to share many similarities to those of other animals (Figure 1F).

Telomere DNA length shortening in bleached samples 
Southern blotting is considered the gold standard technique for telomere DNA length measurement (Aubert, Hills and Lansdorp, 2012). To measure the telomere DNA length of the coral host and its symbiont simultaneously, we adapted the Southern blotting procedure to coral tissue samples and performed sequential hybridizations, first with a probe recognizing the symbiont telomere sequence (TTTAGGG)n and then with a probe recognizing the animal host telomere sequence (TTAGGG)n. Consistent with previous publications on other coral species (Sinclair et al., 2007; Tsuta et al. 2014; Tsuta and Hidaka 2013; Zielke and Bodnar, 2010), colonies of $S$. pistillata revealed terminal DNA fragments hybridizing with a host telomere DNA probe of a mean varying from $3.3 \mathrm{~kb}$ to $5.7 \mathrm{~kb}$ and with the symbionts telomere DNA probe of a mean varying from $3 \mathrm{~kb}$ to $6.2 \mathrm{~kb}$ (Figure $2 \mathrm{~A}$ ).

We induced stress by putting coral colonies in constant darkness (Douglas, A.E., 2003), in two duplicated experiments referred to as D1 and D2 (Supplementary Figure 2). In both experiments S. pistillata colonies were fully bleached after long-term treatment (six months) (Supplementary Figure 2). The telomere analysis was performed on branches of the same colony cut in two and maintained either in control or dark conditions for six months: for D1 (respectively D2), six (respectively four) branches from the bleached half colony and three (respectively two) branches from the control half colony (Figure 2A) were successfully measured twice in Telomere Restriction Fragment assay. The host telomere DNA length was measured reporting the smear mean, median, first quartile (Q1), third quartile (Q3) and interquartile distance (IQ) (Figure 2B, Supplementary Table 1). In both experiments bleached colonies were experiencing shorter telomeres with significantly shorter telomere DNA length mean, median, IQ and Q3 in the host and an absence of signal for the symbionts due to bleached state. However, Q1 measurements of the host telomere DNA length were not significantly different from those of control samples. Thus, the stress triggered by a six month dark incubation of $S$. pistillata shifted the host telomere DNA length distribution towards less long telomeres (Q3) without increasing the short telomeres (Q1) proportion.

\section{Transcriptome profiling of dark-induced bleached S. pistillata}

To unravel a telomere transcriptomic signature of stressed samples, we performed RNA-seq profiling of three control and three bleached branches from the D1 experiment. We identified 862 Differentially Expressed Genes (DEG) (adj. p<0.05), including 602 downregulated and 260 upregulated genes (Figure 3A and Supplementary Table 2). In order to identify the DEGs that could be directly controlled by the dark condition, we compared the transcriptomic profile of bleached samples to a previous transcriptome study performed on S. pistillatacolonies during day and night cycles (Ottaviani et al. 2020) (Figure 3B). We found 31 genes in common and differentially expressed in the same direction between the two sets of DEGs (Figure 3B). We considered that the expression of these genes was controlled by the dark condition rather than the stress triggered by a long period in darkness and leading to a bleached state.

Using the telomere-related interactome reported in the Uniprot database, we identified among the DEGs nine genes that could be involved in telomere metabolism in the stressed samples (Figure 3C). One of the two upregulated genes (Gnmt) was predicted to be Glycine-N methyltransferase involved in DNA methylation, suggesting that the telomere changes triggered by the continuous darkness stress are associated with epigenetic changes. The second upregulated gene (Trpc5 ) is part of a ionic transporter family. Among the downregulated genes, five are known to be involved in cellular growth and tissue remodeling (Cenpf, Plk1, Profilin-2, Mapk6 andPlat) suggesting a link between the telomere shortening occurring in stressed colonies and their reduced growth rate. We also found thatPot2, one of the two protections of telomere shelterin of S. pistillata, was significantly downregulated in bleached samples (Figure 3C). We confirmed, for a subset of genes (seven genes), their significantly expression changes by quantitative PCR analysis both in the samples used for RNA-seq and in biological duplicates using two reference genes with constant expression in control and stressed condition (Trpc2 and Pot1) (Figure 3D, Supplementary Table 2 and 3). We confirmed a trend toward downregulation of Pot2 that is not significant due to a single control branch (S1n4) that was expressing a very low level of Pot2 (Supplementary Table 3).

The most significantly enriched $(p<0.01)$ categories of gene ontology terms (GO terms) among the DEGs were related to metabolism, protein homeostasis, biosynthesis and oxidative stress (GO:0006979, GO:0055114) 
(Figure 4A). When compared with heat induced bleaching transcriptomic studies done on various coral species (Figure 4B-C), we found three common GO terms in the Biological process that are related to growth, proteolysis and macromolecule catabolic process (Figure 4B-C and Supplementary Table 4) (Pinzón, 2015, Seneca and Palumbi. 2015, Traylor-Knowles et al., 2017, Zhou et al., 2017, Li et al., 2021).

\section{Discussion}

This study reveals that a reef coral experiencing a prolonged darkness leading to symbiosis disruption and bleaching exhibits signs of telomere dysfunctions. Notably a shortening of its mean telomere DNA length and a decreased proportion of long telomeres as well as the downregulation of the Pot2 gene encoding a putative subunit of the telomere protective shelterin complex in coral. This finding is a new example of the intimate link between stress response and telomere dysfunction. In the context of the massive coral bleaching events due to the extreme rise of sea water temperatures, these results suggest that long term symbiosis disruption can affect host telomere state and should be taken into account to evaluate the fitness of coral reef survivors.

The enrichment of GO terms linked to oxidative stress response could reflect an increase in the production of reactive oxygen species (ROS), which are likely candidates to cause the observed telomere DNA shortening (Barnes et al., 2019). An increased production of ROS is also the cause of heat-induced bleaching in the wild suggesting a potential telomere dysfunction due to oxidative stress in heat-induced bleaching as what we observed in dark-induced bleaching. Since oxidative stress can be both the cause and the consequence of dysregulated telomeres (Jacome-Burbano and Gilson, 2020), bleaching could, through an increased oxidative environment, create a positive loop accelerating telomere dysfunction and favoring diseases. If oxidative stress is well documented to be a major cause of symbiosis breakdown due to an increase in photosynthetic activity (Lesser, 1997, Dias et al., 2019), the cause of the oxidative stress observed here after six months of darkness is not known. It could result from the maintenance of an oxidative stress response responsible for the initial symbiont loss and/or from a continuous hypoxic condition that can increase ROS production (Lewis et al., 2012).

Like in rodents and the nematode $C$. elegans, the coral genome contains two genes coding for human POT1 orthologs. Interestingly, the second coral Pot gene (named here Pot2 ) seems to have arisen after the divergence of Cnidarian organisms since H. vulgaris exhibits only one Pot1 ortholog. In mammals, Pot1 forms a heterodimeric complex with Tpp1 and has a crucial role in regulating the resection of the telomere DNA overhang and in recruiting telomerase at telomeres (Nandakumar et al., 2012). The host telomere DNA length shortening observed in bleached samples could be explained by the downregulation of Pot2 that would fail to recruit telomerase at long telomeres resulting in an overall decrease in telomere length after six months. Interestingly in another invertebrate that exhibits two Pot proteins, C. elegans telomeres harbor both 5' C- and 3' G-rich overhangs respectively bound by Pot2 and Pot1 (Raices et al., 2008). Since the 5' C-strand overhang can be involved in telomere elongation by recombination (Zhang et al., 2019), it is possible that a function of Pot2 in C. elegans is to regulate telomere elongation by recombination. To investigate whether corals exhibit both types of overhangs and how they are bound by the two Pot proteins could give interesting insights into the mechanisms of telomere DNA regulation in coral.

There are several limits to the conclusion that can be drawn from these results in terms of mechanisms of coral bleaching. First, the bleaching state studied here was triggered by continuous darkness, which is not a classical stressor acting in the wild (Sully et al., 2019; Hughes et al., 2018a). Second, the telomere and transcriptomic response could be more due to long term darkness than to the bleaching per se. Third, the bleached corals were kept alive by external feeding, and the shift from a partial to a complete heterotrophic state modified the coral metabolism and could be responsible for the observed telomere shortening.

The impact of long term darkness was visible in 31 DEGs previously identified in a study on S. pistillata looking at the day-night transcriptome (Figure 3B). When compared with temperature induced bleaching GO 
term enrichment from studies in several coral species (Acropora aculeus, Acropora hyacinthus, Pocillopora damicornis, Orbicella faveolata), it appears that the common ones were first mostly linked to metabolism and second more numerous in the study using the closely related Pocillopora damicornis (Li et al., 2021). It would be of interest to test whether the combined effect of temperature and holobiont disruption can have a short term effect on telomere length regulation. It is important to point out that even under a constant feeding regime, bleached corals experienced a delay in growth, an impaired function found in enriched GO terms of different bleaching studies. Shallow corals have co-evolved with their symbionts and this relationship became mandatory as corals can't properly live without them.

Bleaching recovery is dependent on heat-sensitive to heat-tolerant symbiont turnover, showing the importance of the interplay between symbiont physiology and coral metabolism in survival (Claar et al., 2020). Thus, it would be of interest to investigate whether different symbionts association can lead to different telomere length regulation upon stress.

Nevertheless, the association between telomere DNA shortening,Pot2 downregulation and oxidative stress response in an experimentally controlled darkness situation contributes to our understanding of the telomere changes occurring in coral that have experienced a stress and to the predictions of the impacts of climate change to telomere homeostasis and genome maintenance. Our results suggest that after a bleaching event, the telomere shortening that can persist with time, will affect the long-term health of coral reefs with a higher sensitivity to diseases. Moreover, the inheritability of parental stress bleaching experiences to offspring (Puisay et al., 2020) could be conveyed by telomere dysfunction and impact several generations among the surviving individuals.

\section{Acknowledgement}

We acknowledge the coral culture facility at the Centre Scientifique de Monaco and specifically Dominique Desgre as well as Nathalie Técher and Natacha Segonds. The work in EG lab is supported by the ANR CoralGene and the Inserm cross-cutting program on aging AGEMED. We acknowledge the Bioinformatic and Genomic facility platform of the IRCAN institute as well as Gianni Liti's team for lending its CHEF device to run Southern Blot.

\section{Data Accessibility Statement}

The RNAseq raw data are available with accession number GSE171268 (https://www.ncbi.nlm.nih.gov/geo/query/acc.cgi?acc=GSE171268).

\section{References}

Alexa, A., Rahnenfuhrer, J., \& Lengauer, T. (2006). Improved scoring of functional groups from gene expression data by decorrelating GO graph structure. Bioinformatics , 22 (13), 1600-1607. doi:10.1093/bioinformatics/btl140

Altschul, S. F., Gish, W., Miller, W., Myers, E. W., \& Lipman, D. J. (1990). Basic local alignment search tool. Journal of Molecular Biology , 215 (3), 403-410. doi:10.1016/S0022-2836(05)80360-2

Altschul, Stephen F., Madden, T. L., Schäffer, A. A., Zhang, J., Zhang, Z., Miller, W., \& Lipman, D. J. (1997). Gapped BLAST and PSI-BLAST: A new generation of protein database search programs. Nucleic Acids Research , 25 (17), 3389-3402. doi:10.1093/nar/25.17.3389

Antoine Puisay, Elleaume, N., Fouqueau, L., Lacube, Y., Goiran, C., Sidobre, C., ... Hédouin, L. (2020). Parental bleaching susceptibility leads to differences in larval fluorescence and dispersal potential in Pocillopora 
acuta corals. Marine Environmental Research , 105200. doi:10.1016/j.marenvres.2020.105200

Aubert, G., Hills, M., \& Lansdorp, P. M. (2012). Telomere length measurement_Caveats and a critical assessment of the available technologies and tools. Mutation Research/Fundamental and Molecular Mechanisms of Mutagenesis , 730 (1), 59-67. doi:10.1016/j.mrfmmm.2011.04.003

Barnes, R. P., Fouquerel, E., \& Opresko, P. L. (2019). The impact of oxidative DNA damage and stress on telomere homeostasis. Mech. Ageing Dev. , 177, 37-45. doi:10.1016/j.mad.2018.03.013

Bateson, M. (2016). Cumulative stress in research animals: Telomere attrition as a biomarker in a welfare context? BioEssays ,38 (2), 201-212. doi:https://doi.org/10.1002/bies.201500127

Capella-Gutiérrez, S., Silla-Martínez, J. M., \& Gabaldón, T. (2009). trimAl: A tool for automated alignment trimming in large-scale phylogenetic analyses. Bioinformatics , 25 (15), 1972-1973. doi:10.1093/bioinformatics/btp348

Chen, S., Zhou, Y., Chen, Y., \& Gu, J. (2018). fastp: An ultra-fast all-in-one FASTQ preprocessor. Bioinformatics , 34 (17), i884-i890. doi:10.1093/bioinformatics/bty560

Claar, D. C., Starko, S., Tietjen, K. L., Epstein, H. E., Cunning, R., Cobb, K. M., .. Baum, J. K. (2020). Dynamic symbioses reveal pathways to coral survival through prolonged heatwaves. Nature Communications , 11 (1), 6097. doi:10.1038/s41467-020-19169-y

Danovaro, R., Bongiorni, L., Corinaldesi, C., Giovannelli, D., Damiani, E., Astolfi, P., ... Pusceddu, A. (2008). Sunscreens cause coral bleaching by promoting viral infections. Environ. Health Perspect. , 116 (4), 441-447. doi:10.1289/ehp.10966

Darriba, D., Posada, D., Kozlov, A. M., Stamatakis, A., Morel, B., \& Flouri, T. (2020). ModelTest-NG: A New and Scalable Tool for the Selection of DNA and Protein Evolutionary Models. Molecular Biology and Evolution , 37 (1), 291-294. doi:10.1093/molbev/msz189

Dias, M., Ferreira, A., Gouveia, R., Madeira, C., Jogee, N., Cabral, H., .. Vinagre, C. (2019). Longterm exposure to increasing temperatures on scleractinian coral fragments reveals oxidative stress. Marine Environmental Research , 150 , 104758. doi:10.1016/j.marenvres.2019.104758

Douglas, A. E. (2003). Coral bleaching-how and why? Marine Pollution Bulletin , 46 (4), 385-392. doi:10.1016/S0025-326X(03)00037-7

Downs, C. A., McDougall, K. E., Woodley, C. M., Fauth, J. E., Richmond, R. H., Kushmaro, A., ... Kramarsky-Winter, E. (2013). Heat-Stress and Light-Stress Induce Different Cellular Pathologies in the Symbiotic Dinoflagellate during Coral Bleaching. PLoS ONE , 8 (12), e77173. doi:10.1371/journal.pone.0077173

Downs, Craig A., Kramarsky-Winter, E., Martinez, J., Kushmaro, A., Woodley, C. M., Loya, Y., \& Ostrander, G. K. (2009). Symbiophagy as a cellular mechanism for coral bleaching. Autophagy , 5 (2), $211-216$. doi:10.4161/auto.5.2.7405

Edler, D., Klein, J., Antonelli, A., \& Silvestro, D. (2020). RaxmlGUI 2.0: A graphical interface and toolkit for phylogenetic analyses using RAxML. Methods in Ecology and Evolution. Retrieved fromhttps://doc.rero.ch/record/329733

Furla, P., Allemand, D., Shick, J. M., Ferrier-Pagès, C., Richier, S., Plantivaux, A., .. Tambutté, S. (2005). The Symbiotic Anthozoan: A Physiological Chimera between Alga and Animal. Integrative and Comparative Biology , 45 (4), 595-604. doi:10.1093/icb/45.4.595

Gardner, S. G., Raina, J.-B., Nitschke, M. R., Nielsen, D. A., Stat, M., Motti, C. A., ... Petrou, K. (2017). A multi-trait systems approach reveals a response cascade to bleaching in corals. BMC Biology ,15 . doi:10.1186/s12915-017-0459-2 
Gille, C., Fähling, M., Weyand, B., Wieland, T., \& Gille, A. (2014). Alignment-Annotator web server: Rendering and annotating sequence alignments. Nucleic Acids Research , 42 (Web Server issue), W3-W6. doi:10.1093/nar/gku400

Gilson, E., \& Géli, V. (2007). How telomeres are replicated.Nature Reviews Molecular Cell Biology , 8 (10), 825-838. doi: 10.1038/nrm2259

Giraud-Panis, M.-J., Pisano, S., Benarroch-Popivker, D., Pei, B., Le Du, M.-H., \& Gilson, E. (2013). One Identity or More for Telomeres? Frontiers in Oncology , 3 . doi:10.3389/fonc.2013.00048

Hughes, T. P., Anderson, K. D., Connolly, S. R., Heron, S. F., Kerry, J. T., Lough, J. M., ... Wilson, S. K. (2018). Spatial and temporal patterns of mass bleaching of corals in the Anthropocene.Science , 359 (6371), 80-83. doi:10.1126/science.aan8048

Hughes, T. P., Kerry, J. T., Baird, A. H., Connolly, S. R., Dietzel, A., Eakin, C. M., .. Torda, G. (2018). Global warming transforms coral reef assemblages. Nature , 556 (7702), 492-496. doi:10.1038/s41586-0180041-2

Jacome Burbano, M. S., \& Gilson, E. (2020). Long-lived post-mitotic cell aging: Is a telomere clock at play? Mechanisms of Ageing and Development, 189 , 111256. doi:10.1016/j.mad.2020.111256

Kanehisa, M. (2000). KEGG: Kyoto Encyclopedia of Genes and Genomes.Nucleic Acids Research , 28 (1), 27-30. doi:10.1093/nar/28.1.27

Katoh, K., \& Standley, D. M. (2013). MAFFT Multiple Sequence Alignment Software Version 7: Improvements in Performance and Usability.Molecular Biology and Evolution , 30 (4), 772-780. doi: $10.1093 / \mathrm{molbev} / \mathrm{mst010}$

Krämer, A., Green, J., Pollard, J., \& Tugendreich, S. (2014). Causal analysis approaches in Ingenuity Pathway Analysis.Bioinformatics , 30 (4), 523-530. doi:10.1093/bioinformatics/btt703

Kushmaro, A., Loya, Y., Fine, M., \& Rosenberg, E. (1996). Bacterial infection and coral bleaching. Nature , 380 (6573), 396-396. doi:10.1038/380396a0

Lange, T. de. (2005). Shelterin: The protein complex that shapes and safeguards human telomeres. Genes \& Development , 19 (18), 2100-2110. doi:10.1101/gad.1346005

Lesser, M. P. (1997). Oxidative stress causes coral bleaching during exposure to elevated temperatures. Coral Reefs , 16 (3), 187-192. doi:10.1007/s003380050073

Letunic, I., \& Bork, P. (2019). Interactive Tree Of Life (iTOL) v4: Recent updates and new developments. Nucleic Acids Research ,47 (W1), W256-W259. doi:10.1093/nar/gkz239

Lewis, K. N., Andziak, B., Yang, T., \& Buffenstein, R. (2012). The Naked Mole-Rat Response to Oxidative Stress: Just Deal with It.Antioxidants $\&$ Redox Signaling , 19 (12), 1388-1399. doi:10.1089/ars.2012.4911

Li, J., Long, L., Zou, Y., \& Zhang, S. (2021). Microbial community and transcriptional responses to increased temperatures in coral Pocillopora damicornis holobiont. Environmental Microbiology , 23 (2), 826-843. doi:https://doi.org/10.1111/1462-2920.15168

López-Otín, C., \& Kroemer, G. (2021). Hallmarks of Health. Cell ,184 (1), 33-63. doi:10.1016/j.cell.2020.11.034

Love, M. I., Huber, W., \& Anders, S. (2014). Moderated estimation of fold change and dispersion for RNA-seq data with DESeq2. Genome Biology , 15 (12), 550. doi:10.1186/s13059-014-0550-8

Lyndby, N. H., Holm, J. B., Wangpraseurt, D., Grover, R., Rottier, C., Kühl, M., \& Ferrier-Pagès, C. (2020). Effect of temperature and feeding on carbon budgets and $\mathrm{O} 2$ dynamics in Pocillopora damicornis. Marine Ecology Progress Series , 652, 49-62. doi:10.3354/meps13474 
Ma, S., Upneja, A., Galecki, A., Tsai, Y.-M., Burant, C. F., Raskind, S., .. Gladyshev, V. N. (2016). Cell culture-based profiling across mammals reveals DNA repair and metabolism as determinants of species longevity. ELife, 5, e19130. doi:10.7554/eLife.19130

Maor-Landaw, K., \& Levy, O. (2016). Gene expression profiles during short-term heat stress; branching vs. Massive Scleractinian corals of the Red Sea. PeerJ , 4 , e1814. doi:10.7717/peerj.1814

Monaghan, P. (2014). Organismal stress, telomeres and life histories. Journal of Experimental Biology , 217 (1), 57-66. doi:10.1242/jeb.090043

Nandakumar, J., Bell, C. F., Weidenfeld, I., Zaug, A. J., Leinwand, L. A., \& Cech, T. R. (2012). The TEL patch of telomere protein TPP1 mediates telomerase recruitment and processivity. Nature,492 (7428), 285-289. doi:10.1038/nature11648

Ottaviani, A., Eid, R., Zoccola, D., Pousse, M., Dubal, J.-M., Barajas, E., ... Allemand, D. (2020). Longevity strategies in response to light in the reef coral Stylophora pistillata. Sci. Rep. ,10 (1), 19937. doi:10.1038/s41598-020-76925-2

Patro, R., Duggal, G., Love, M. I., Irizarry, R. A., \& Kingsford, C. (2017). Salmon provides fast and biasaware quantification of transcript expression. Nature Methods , 14 (4), 417-419. doi:10.1038/nmeth.4197

Pinzón, J. H., Kamel, B., Burge, C. A., Harvell, C. D., Medina, M., Weil, E., \& Mydlarz, L. D. (2015). Whole transcriptome analysis reveals changes in expression of immune-related genes during and after bleaching in a reef-building coral. Royal Society Open Science,2 (4). doi:10.1098/rsos.140214

Poulet, A., Pisano, S., Faivre-Moskalenko, C., Pei, B., Tauran, Y., Haftek-Terreau, Z., .. Giraud-Panis, M.-J. (2012). The N-terminal domains of TRF1 and TRF2 regulate their ability to condense telomeric DNA. Nucleic Acids Research , 40 (6), 2566-2576. doi:10.1093/nar/gkr1116

Raices, M., Verdun, R. E., Compton, S. A., Haggblom, C. I., Griffith, J. D., Dillin, A., \& Karlseder, J. (2008). C. elegans Telomeres Contain G-Strand and C-Strand Overhangs that Are Bound by Distinct Proteins. Cell , 132 (5), 745-757. doi:10.1016/j.cell.2007.12.039

Reef, R., Dunn, S., Levy, O., Dove, S., Shemesh, E., Brickner, I., ... Hoegh-Guldberg, O. (2009). Photoreactivation is the main repair pathway for UV-induced DNA damage in coral planulae. Journal of Experimental Biology , 212 (17), 2760-2766. doi:10.1242/jeb.031286

Rosenberg, E., Kushmaro, A., Kramarsky-Winter, E., Banin, E., \& Yossi, L. (2009). The role of microorganisms in coral bleaching. The ISME Journal , 3 (2), 139-146. doi:10.1038/ismej.2008.104

Seneca, F. O., \& Palumbi, S. R. (2015). The role of transcriptome resilience in resistance of corals to bleaching. Molecular Ecology , 24 (7), 1467-1484. doi:10.1111/mec.13125

Sinclair, C. S., Richmond, R. H., \& Ostrander, G. K. (2007). Characterization of the telomere regions of scleractinian coral, Acropora surculosa. Genetica , 129 (3), 227-233. doi:10.1007/s10709-006-0001-x

Sully, S., Burkepile, D. E., Donovan, M. K., Hodgson, G., \& van Woesik, R. (2019). A global analysis of coral bleaching over the past two decades. Nature Communications , 10 (1), 1264. doi:10.1038/s41467-01909238-2

Tian, X., Firsanov, D., Zhang, Z., Cheng, Y., Luo, L., Tombline, G., .. Gorbunova, V. (2019). SIRT6 Is Responsible for More Efficient DNA Double-Strand Break Repair in Long-Lived Species. Cell ,177 (3), 622-638.e22. doi:10.1016/j.cell.2019.03.043

Traut, W., Szczepanowski, M., Vítková, M., Opitz, C., Marec, F., \& Zrzavý, J. (2007). The telomere repeat motif of basal Metazoa.Chromosome Research . doi:10.1007/s10577-007-1132-3

Traylor-Knowles, N., Rose, N. H., Sheets, E. A., \& Palumbi, S. R. (2017). Early Transcriptional Responses during Heat Stress in the Coral Acropora hyacinthus. The Biological Bulletin , 232 (2), 91-100. 
doi:10.1086/692717

Tsuta, H., \& Hidaka, M. (2013). Telomere length of the colonial coral Galaxea fascicularis at different developmental stages. Coral Reefs , 32 (2), 495-502. doi:10.1007/s00338-012-0997-6

Tsuta, Hiroki, Shinzato, C., Satoh, N., \& Hidaka, M. (2014). Telomere Shortening in the Colonial Coral Acropora digitifera During Development. Zoological Science , 31 (3), 129-134. doi:10.2108/zsj.31.129

Voolstra, C. R., Li, Y., Liew, Y. J., Baumgarten, S., Zoccola, D., Flot, J.-F., .. . Aranda, M. (2017). Comparative analysis of the genomes of Stylophora pistillata and Acropora digitifera provides evidence for extensive differences between species of corals. Scientific Reports , 7 (1). doi:10.1038/s41598-017-17484-x

Ye, J., Renault, V. M., Jamet, K., \& Gilson, E. (2014). Transcriptional outcome of telomere signalling. Nature Reviews Genetics ,15 (7), 491-503. doi:10.1038/nrg3743

Young, A. J. (2018). The role of telomeres in the mechanisms and evolution of life-history trade-offs and ageing. Philosophical Transactions of the Royal Society B: Biological Sciences ,373 (1741), 20160452. doi:10.1098/rstb.2016.0452

Young, R. C., Orben, R. A., Will, A. P., \& Kitaysky, A. S. (2017). Relationship between telomere dynamics and movement and behavior during winter in the thick-billed murre. Marine Ecology Progress Series ,578 , 253-261. doi:10.3354/meps12022

Zhang, T., Zhang, Z., Shengzhao, G., Li, X., Liu, H., \& Zhao, Y. (2019). Strand break-induced replication fork collapse leads to C-circles, C-overhangs and telomeric recombination. PLoS Genetics, 15 (2), e1007925. doi:10.1371/journal.pgen.1007925

Zhou, Z., Wu, Y., Zhang, C., Li, C., Chen, G., Yu, X., .. Huang, B. (2017). Suppression of NF- $x$ B signal pathway by NLRC3-like protein in stony coral Acropora aculeus under heat stress. Fish E Shellfish Immunology , 67 , 322-330. doi:10.1016/j.fsi.2017.06.027

Zielke, S., \& Bodnar, A. (2010). Telomeres and telomerase activity in scleractinian corals and Symbiodinium spp. The Biological Bulletin , 218 (2), 113-121.

\section{Figure legends}

Figure 1 : Stylophora pistillata shelterin proteins and telomerase phylogenetic trees. Phylogenetic trees based on protein sequences looking for human orthologs of S. pistillata shelterin subunits.(A) RAP1 phylogenetic tree supports the presence of a conserved protein between cnidarians (B) TRF phylogenetic tree supports the presence of only one TRF shelterin in cnidarians and two in vertebrates (TRF1 and TRF2) (C) TERT phylogenetic tree supports the presence of the telomerase holoenzyme subunit in cnidarians conserved protein between cnidarians (D) POT1 phylogenetic tree supports the presence of 2 POT1 shelterin in corals (S. pistillata , A. digitifera ,P. damicornis ) such as in C. elegans (POT1 and POT2) (E) TPP1phylogenetic tree supports the presence of a conserved protein between cnidarians (F) Scheme of Homo sapiens shelterin complex and telomerase subunits and of Stylophora psitillata shelterin and telomerase subunit found in this work. (G) Localization of conserved domains (OB fold and Pot1-Cterminal domain) in human Pot1 and S. pistillata Pot1 and Pot2.

Figure 2 : Stylophora pistillata dark induced bleaching telomere length measured by Telomeric Restriction Fragment assay. (A) Telomeric Restriction Fragment assay from D1 experiment, left panel is showing the membrane labelled with the (TTAGGG)n coral DNA telomeric sequence complementary probe and right panel is the membrane labelled with the radioactive (TTTAGGG)n symbionts DNA telomeric sequence complementary probe. 5 control branches and 6 bleached branches are displayed. Bleached branches after 6 months of darkness exposure exhibits a shorter telomeric smear signal with the (TTAGGG)n coral telomere probe labelling and no signal with the (TTTAGGG)n symbiont telomere probe (B) Boxplot of (TTAGGG)n coral telomere smear measurements in kilobases (kb), IQ (interquartile distance between first 
quartile Q1 and third quartile Q3), Mean telomere length and median telomere length between bleached and control samples from experiment D1 (circles) and D2 (triangles), TL IQ, Mean, Median and Q3 was significantly shorter in bleached conditions (respectively $p$ value $=0.0338,0.0338,0.498$ and 0.0338 ) except for the Q1 that was not significantly different between the two conditions ( $p$ value $=0.114)$.

Figure 3 : Stylophora pistillata bleaching RNA sequencing results. (A) Volcano plot of significantly differentially expressed genes in bleached condition (pvalue $<0.05)$ of the RNA sequencing of 3 branches ( $\mathrm{n}=$ nubbins) of control S. pistillatacolony S1 and dark induced bleached colony (S1B). (B) Common differentially expressed gene in bleached transcriptome (left) and in night and day transcriptome (right) from (Ottaviani et al., 2020), log2(fold change) is displayed with colors (pink for negatively DEG and green for positively DEG). (C) Significant differential expression expressed in Log2(FoldChange) of telomere related genes (Gene names) in bleached condition $(\mathrm{p}<0.05)$. (D) Fold change log of differentially expressed genes (Apod, CENPF, Profilin2, MAPK6, Pot2, Plk1, Ddx21, Trpc5, Gnmt, PXDN) performed by quantitative PCR using two different control genes (Pot1 and Trpc2) and by RNA sequencing. Statistics were performed using student tests on log results of Pfaffl equation (Supplementary table 3).

Figure 4 : Gene Ontology enrichment in bleached coral samples.(A) Biological process Go-term significant enrichment in dark induced bleached samples. (B) Venn diagramm of Go-terms compared with previous transcriptomic studies performed on heat induced bleaching samples. Three biological process Goterms of our study were found in one of the four others as well as 1 cellular component and 1 molecular function one. (C) Common Go-terms between our study and the investigated transcriptomic studies. We included the study from (Zhou et al., 2017) but due to a total absence of common Go-terms this crossing was not displayed.

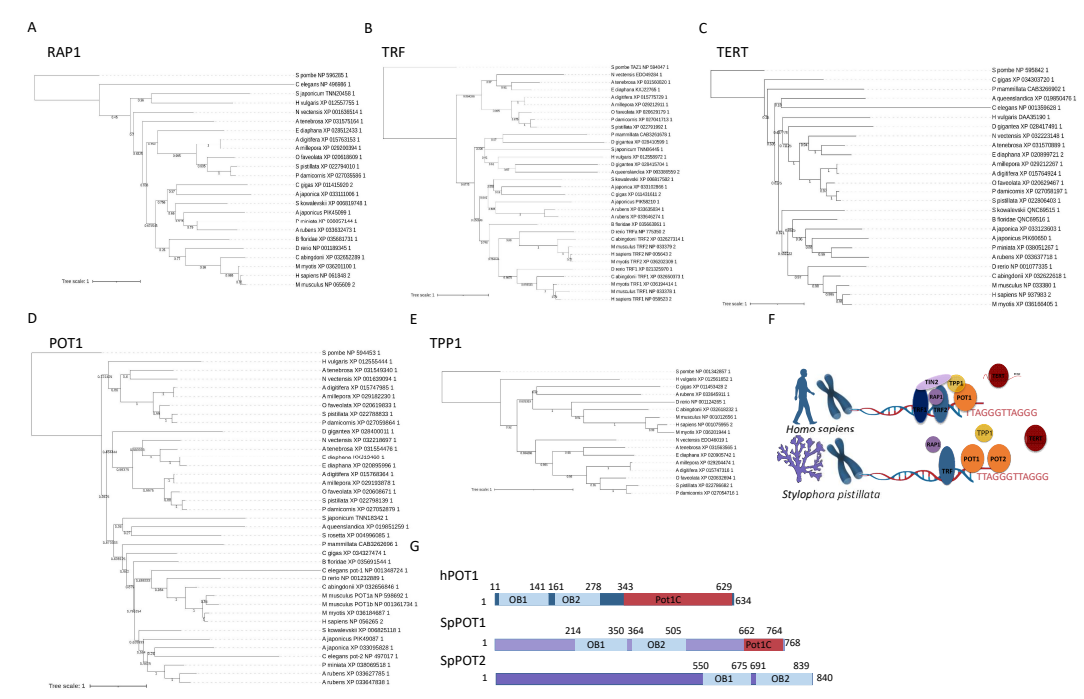



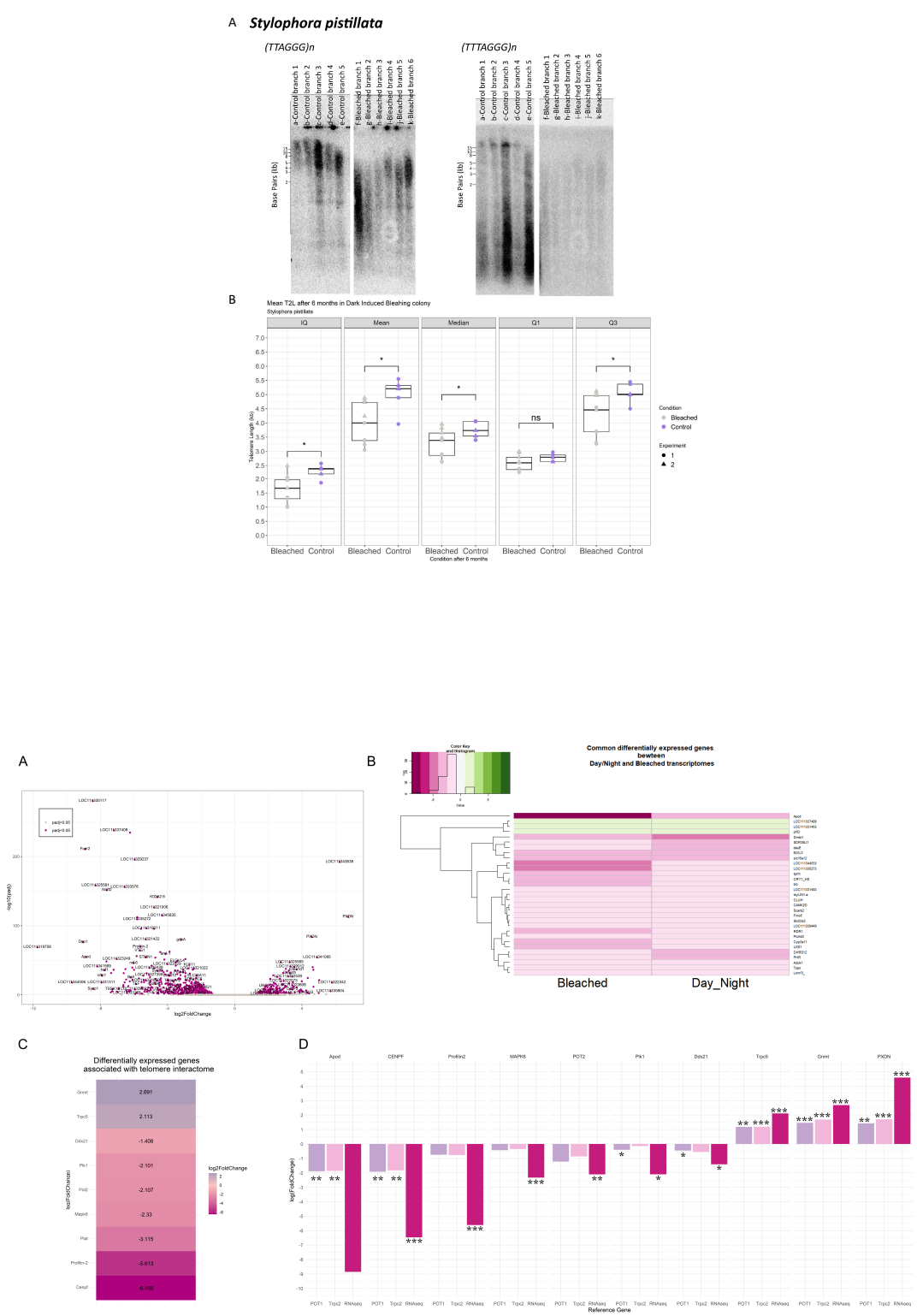
A

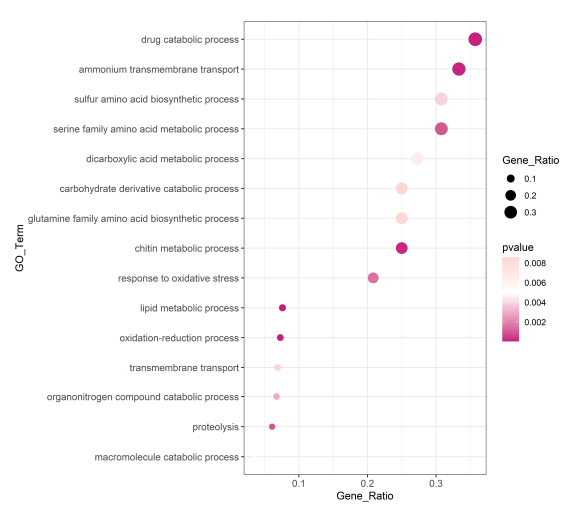

c

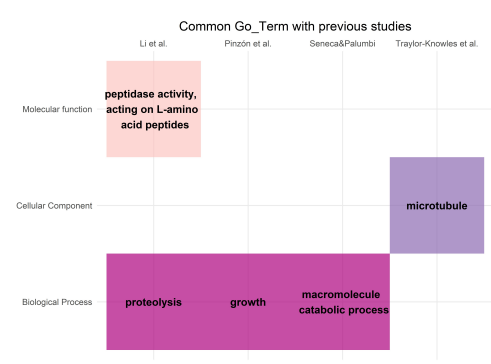

B
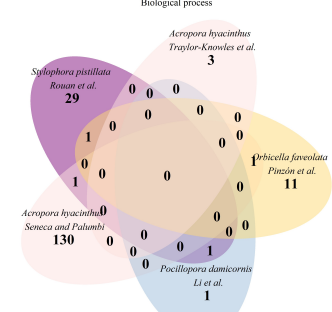

Letal.
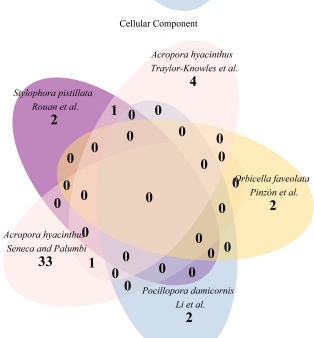

volues

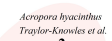

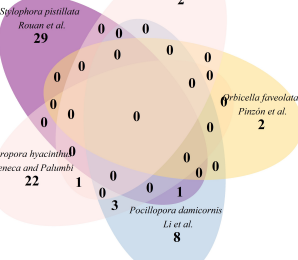

